\title{
ARTÍCULO ORIGINAL: Reconstrucción de lengua: Evidencia reciente y experiencia en el Hospital San Juan de Dios (HSJD), entre octubre de 2010 y junio de 2011 .
}

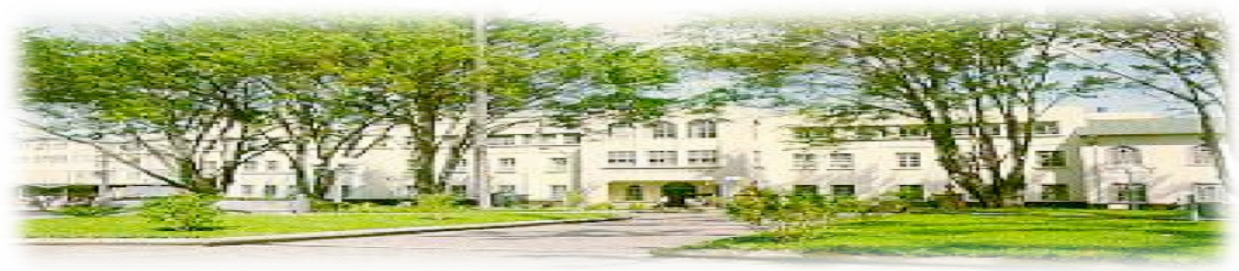

Haspital San Juan de Dias. San Jasé. Costa Rica. Fundada en 1845

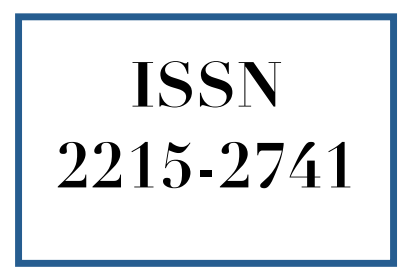

Recibido: $\quad$ 05/05/2013

Aceptado: $\quad 17 / 07 / 2013$

Daniel Sancho Camacho' Josías Juantá Castro ${ }^{2}$

${ }^{1}$ Médico General. Sección de Oncología. Hospital San Juan de Dios.

${ }^{2}$ Especialista en Cirugía Oncológica. Sección de Oncología. Hospital San Juan de Dios.

\section{RESUMEN}

La reconstrucción post resección de tumores de lengua es uno de los más complejos problemas para resolver en la oncología de cabeza y cuello, debido a la gran cantidad de abordajes propuestos y a la dificultad intrínseca que presenta la lengua como órgano importante para el habla y la deglución. Por lo tanto, la reconstrucción de ésta debe cumplir con el reto de alcanzar resultados funcionales, que se aproximen al estado previo del paciente. El colgajo libre radial de antebrazo, descrito en la literatura y utilizado en nuestro centro, se proyecta como el abordaje ideal para alcanzar estos resultados, sin embargo existe evidencia con el uso de otros tipos de colgajos, por ende la experiencia del cirujano es importante en la decisión del manejo. Al revisar la experiencia en el HSJD se encuentra que ésta se aproxima a la realidad mundial descrita en la literatura, tanto en los resultados como en la patología encontrada.

\section{PALABRAS CLAVE}

Cáncer de lengua. Reconstrucción de lengua. Colgajo libre radial de antebrazo. Microcirugía.

\begin{abstract}
Reconstruction after a resection of a tongue's tumor is one the most complex problems in head and neck oncology, it happens not only because of the multiple variety of surgical approaches but also on the intrinsic difficulty of the tongue due to the important role that the organ plays in both, speech and swallowing. Thus, the reconstruction should attempt to reestablish the pre surgical
\end{abstract}


status of the patient as good as posible. The radial free forearm flap, described in the literature and used in our center, comes forth as the ideal approach to accomplish this results, nonetheless, there is evidence regarding other types of flap reconstruction, hence the important role that the experience of the surgical team has in decision making. Reviewing the experience in the Hospital San Juan de Dios, we realized that it approaches the worldwide experience in both pathology and results.

\section{KEY WORDS}

Tongue reconstruction. Radial free forearm flap. Microsurgery.

\section{INTRODUCCIÓN}

La reconstrucción luego de la resección de un tumor y entre éstos, los tumores malignos de lengua, continúa siendo uno de los problemas más complicados de resolver en oncología quirúrgica de cabeza y cuello. La lengua tiene un rol crítico en aspectos tan importantes de nuestra vida diaria como lo son la deglución y el habla, por lo tanto una reconstrucción exitosa debe contar con rehabilitación funcional, que se aproxime lo más posible al estado previo del paciente, luego de una resección mayor de lengua.

En el pasado, la mayoría de defectos orales se cerraban de forma primaria utilizando colgajos locales o regionales, fuesen cutáneos o mucosos. Esta tendencia cambió inicialmente en 1979, cuando Aryan desarrolló el colgajo miocutáneo de músculo pectoral mayor ${ }^{(1)}$ el cual por la próxima década se convirtió en el "caballo de batalla" para los cirujanos de cabeza y cuello. Desde entonces, la mayor contribución a la reconstrucción de cabeza y cuello en las últimas tres décadas ha sido la transferencia de tejido libre microvascular. Esta técnica permite a los cirujanos mucho mejor control local y mejores resultados funcionales.

Por ende, los colgajos libres microvasculares son considerados recientemente como la técnica ideal para la reconstrucción, luego de la remoción de un tumor de cabeza y cuello. A pesar de que existen múltiples técnicas que utilizan este tipo de acercamiento, en el presente estudio se revisa- rá la experiencia en el HSJD, entre octubre de 2010 a junio de 2011.

La lengua es el sitio más común de cáncer dentro de la boca, siendo los 2/3 anteriores del borde lateral de ésta el sitio de más lesiones malignas ${ }^{(2)}$. En casos de carcinoma avanzado de lengua, la terapia agresiva ya sea glosectomía subtotal o hemi-glosectomía, cuando son oncológicamente posibles, aumentan el potencial de mejores resultados funcionales. Existen muchos tipos de acercamiento quirúrgico para este importante reto, sin embargo ninguno ha podido generar una neolengua con movilidad y sensibilidad a la vez $^{(2-3)}$.

El colgajo libre radial de antebrazo (RFFF por sus siglas en inglés; radial free forearm flap), descrito primero en China en 1981 por Yang et $a l .^{(4)}$, de ahí el término "colgajo Chino", fue introducido para reconstrucción intraoral por Soutar et al. ${ }^{(5)}$ en 1983 y desde entonces se ha utilizado para reconstrucciones inmediatas de lengua en resecciones por cáncer ${ }^{(6)}$. Este colgajo cumple con una serie de ventajas en cuanto a la reconstrucción intraoral, las cuales se discutirán más adelante.

La revisión de la literatura actual muestra una tendencia creciente hacia la reconstrucción con colgajos corregidos para la anatomía y con reinervación sensitiva para obtener resultados funcionales superiores, además de la revisión comparativa entre diferentes técnicas de colgajos libres. Aún con toda la evidencia actual, la escogencia del abordaje quirúrgico recae predominantemente en la experiencia del cirujano y los consensos internacionales por parte de expertos en la materia.

\section{Principios en la reconstrucción de la lengua}

Los tumores primarios de la lengua pueden ocurrir en cualquiera de sus partes, sin embargo ocurren principalmente en los bordes laterales, Borggreven et al. ${ }^{(7)}$ lograron demostrar que ocurren con igual frecuencia en ambos lados de la lengua y que la mayoría de se presentaban de forma unilateral, sin cruzar la línea media (73\%).

De los tumores de lengua más frecuentes, el de tipo carcinoma epidermoide de células escamosas es el principal. En muchas ocasiones el control de esta enfermedad requerirá un manejo 
quirúrgico agresivo, para lograr márgenes libres de enfermedad y así minimizar la recurrencia. Dichos márgenes se consideran adecuados con 1.5 a $2 \mathrm{~cm}^{(8)}$.

El planteamiento en cualquier reconstrucción post resección de tumores de lengua debe ser evaluado de forma escalonada, ya que así como las lesiones pueden ir de pequeñas y simples a grandes y complejas, así mismo se escoge el tipo de acercamiento para el cierre del defecto posterior, el cual pueden ir desde segunda intención, cierre primario o injerto, hasta procedimientos complejos como colgajos regionales, colgajos rotacionales y colgajos libres microvasculares. Es por este motivo que la experiencia del cirujano es importante antes de decidir el abordaje, tomando en cuenta el sitio y la extensión de la lesión $^{(9)}$.

La preservación de la forma y la función de la lengua es esencial para un adecuado resultado post quirúrgico. En un estudio sobre cierre primario en defectos, Monsier et al. ${ }^{(10)}$ demostraron que las reconstrucciones que más se aproximan a la biomecánica de la lengua original presentaron mayor adaptación cortical de la neolengua.

Al dividir la lengua, la parte oral de ésta es responsable de la manipulación oral del alimento y la parte basal es responsable por el inicio de la fase oral del mecanismo de la deglución. Esta división se hace a nivel de las papilas caliciformes. Por ende se debe realizar el mayor esfuerzo en reconstruir la cavidad oral y que permita el más amplio rango de movimiento y coordinación.

La lengua residual luego de la resección puede aproximar el colgajo al paladar y la faringe, por eso entre más lengua residual podamos conservar, la manipulación oral de los alimentos y la deglución también mejorarán.

Al introducir un tejido delgado y pliable, la RFFF hace que la lengua mantenga su máxima capacidad móvil, aumentando la inteligibilidad en la articulación de las palabras ${ }^{(11)}$.

\section{Reconstrucción de Lengua}

\section{Tendencias recientes en técnicas de colgajos.}

El RFFF continúa encontrando gran aceptación en el manejo reconstructivo de defectos mayores post glosectomía. Este colgajo es delgado, con gran capacidad elástica y con vasos de calibre adecuado para realizar microanatomosis vascular. Dentro de sus otras ventajas se encuentra una anatomía del colgajo consistente, una aceptable comorbilidad del sitio donador y la habilidad de tomar el colgajo al mismo tiempo que se extirpa el tumor, utilizando dos equipos. Recientemente se ha reportado refinamientos de esta técnica, los cuales incluyen combinación de RFFF con colgajos locales para defectos mayores, la restauración de la sensibilidad a través de anastomosis nerviosa entre el nervio antebraquial cutáneo lateral y el nervio lingual, además de modificaciones en el diseño del colgajo, para poder cubrir un defecto tridimensional, como es la hemi-glosectomía ${ }^{(9)}$.

Biglioli et al. ${ }^{(12)}$ estudiaron el rol que cumple la re-inervación sensorial en una serie de 38 pacientes, los cuales iban a ser sometidos a reconstrucción con colgajo libre radial de antebrazo. De estos pacientes al 50\% (18 pacientes) se les realizó la anastomosis. El procedimiento fue una anastomosis término-terminal con el nervio lingual, utilizando goma de fibrina y dos puntos de sutura en el perineuro con Prolene 10-0. En este estudio no se reporta pérdida del colgajo en ninguno de sus pacientes y sí una mayor recuperación sensorial en los pacientes re-inervados. Esto podría explicarse gracias al gran área que ocupa la lengua en el homúnculo motor.

\section{Reconstrucción de lengua Resultados Funcionales}

Durante mucho tiempo se creyó que un resultado adecuado en la reconstrucción de lengua era una buena cicatrización y que el colgajo sobreviviera, sin embargo, en la actualidad la idea de que un adecuado resultado funcional es de mayor importancia ha venido en aumento. La compleja coordinación muscular para los mecanismos de deglución y habla, requieren de nervios espinales, craneales y propioceptores en el órgano como tal. $\mathrm{Si}$ algunos de estos mecanismos falla, el resultado puede ser disfagia, aspiración o pobre inteligibilidad del habla ${ }^{(9)}$.

Múltiples estudios recientes enfocan sus esfuerzos en identificar el rol que cumple la reinervación en los colgajos de lengua y como ésta podría ayudar en la mejoría de los resultados funcionales.

La anastomosis neural puede reducir la tasa de degeneración Walleriana y así prevenir la atrofia 
del colgajo ${ }^{(13)}$. Este último punto también toma en cuenta el gran área de representación de la lengua en el homúnculo sensorial, para explicar el éxito de la anastomosis, como fue demostrado por estudios de discriminación de dos puntos en el colgajo libre radial de antebrazo ${ }^{(12)}$. Biglioli et al. presentaron una serie de casos de reconstrucciones de lengua utilizando RFFF con conservación de la función del nervio lingual, por medio de anastomosis con el nervio ante-braquial cutáneo lateral.

Reportes subjetivos sobre la capacidad del habla por parte de los pacientes demostraron una mayor satisfacción en los pacientes con colgajos neuro-fascio-cutáneos que con los pacientes sin la conservación sensitiva $(78 \%$ vs $43 \%$ respectivamente). Una evaluación objetiva realizada con el test Fanzago, de articulación del lenguaje, también demostró la obtención de mejores resultados en los pacientes con re-inervación (44\%) en comparación con los pacientes sin ésta (29\%).

En un estudio realizado por $\mathrm{Su}$ et al. que comparó los resultados funcionales de la reconstrucción post hemi-glosectomía utilizando colgajo de pectoral mayor vs RFFF (sin re-inervación), se obtuvo mejores resultados en los scores en cuanto a la capacidad del habla en los pacientes con RFFF, que en los pacientes con colgajo de pectoral mayor (6.23 vs 4.21 respectivamente, para una $\mathrm{p}=0.000$ ). En cuanto a la deglución, no hubo diferencia estadísticamente significativa entre los dos métodos reconstructivos $(\mathrm{p}=0.061)^{(11)}$.

\section{RESULTADOS}

\section{Reconstrucción de lengua Experiencia el en Hospital San Juan de Dios}

Entre diciembre del 2010 y junio del 2011 se realizó dos hemi-glosectomías por carcinoma de lengua, con reconstrucción con RFFF en el HSJD. Ambos (100\%) se hallaban en estadios $\mathrm{T}_{2} \mathrm{~N}_{0}$ y contaban con reporte de patología de carcinoma epidermoide bien diferenciado. Estos estadiajes se realizaron de acuerdo a los criterios del American Committee on Cancer Staging System, $6^{\text {a }}$ edición.

En los dos casos se obtuvo márgenes libres de enfermedad en la pieza final, además de cero ganglios positivos en los reportes patológicos.
Ninguno de los dos pacientes requirió tratamiento adyuvante con radioterapia ya que no cumplían con los requisitos para adyuvancia. (Tabla $1)$.

Tabla 1. Criterios para recibir adyuvancia

\begin{tabular}{|l|}
\hline Tamaño del tumor $>\mathrm{T}_{3}$ \\
\hline Márgenes quirúrgicos positivos \\
\hline Ganglios positivos \\
\hline Extensión extracapsular \\
\hline
\end{tabular}

Fuente: Head Neck. 2004;47:275-361.

Aunque se considera a la radioterapia y la cirugía como la mejor opción potencial para el tratamiento primario del cáncer de lengua, la mayoría de estos carcinomas se tratan de forma quirúrgica únicamente ${ }^{(15)}$.

Los dos pacientes recibieron hemi-glosectomía en el sitio primario y cierre con injerto en la zona donadora.

Las características de los pacientes se evalúan en la Tabla 2.

Tabla 2. Características de los pacientes del HSJD.

\begin{tabular}{|c|c|c|}
\hline Edad & 72 & 50 \\
\hline Género & Masc & Masc \\
\hline $\begin{array}{c}\text { Clasificación } \\
\mathbf{T} \\
\mathbf{N}\end{array}$ & $\begin{array}{l}2 \\
0\end{array}$ & $\begin{array}{l}2 \\
0\end{array}$ \\
\hline Estadio & I & I \\
\hline Grado & Bien diferenciado & Bien diferenciado \\
\hline Márgenes & Neg & Neg \\
\hline $\begin{array}{l}\text { Invasión } \\
\text { linfovascular }\end{array}$ & Neg & Neg \\
\hline Adyuvancia & No & No \\
\hline Cirugía & Hemiglosectomía & Hemiglosectomía \\
\hline $\begin{array}{l}\text { Disección } \\
\text { de cuello }\end{array}$ & No & No \\
\hline
\end{tabular}




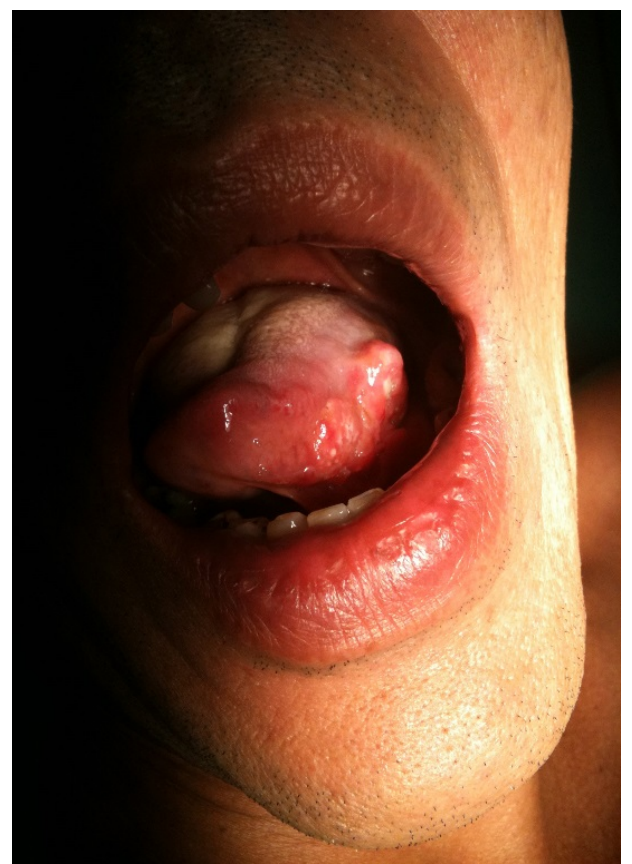

Imagen1. Tumor de lengua previo a la cirugía

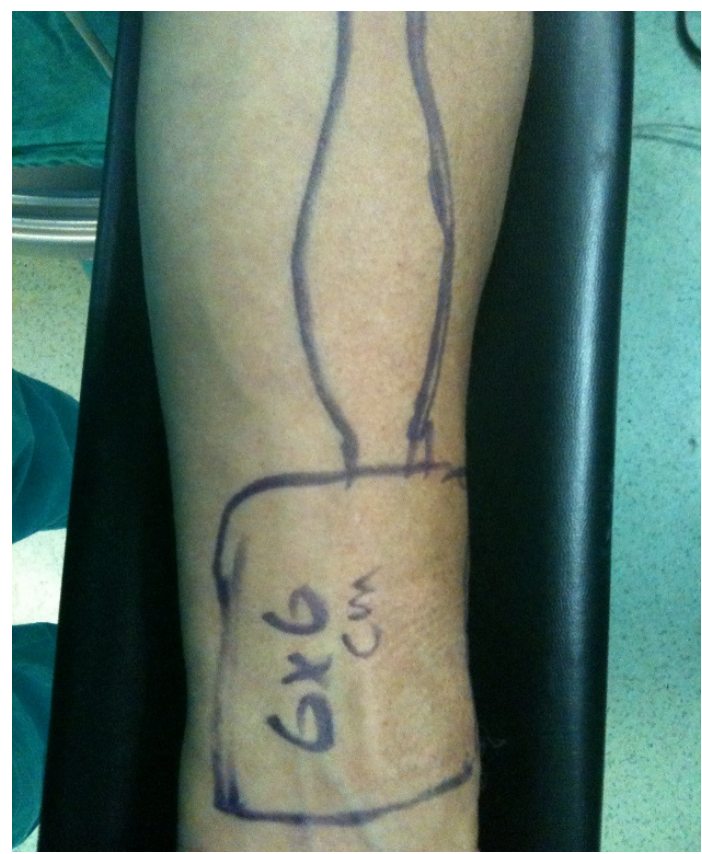

Imagen 2. Sitio del antebrazo con la marca de la línea de corte para el colgajo

En cuanto a la valoración de los resultados, una evaluación subjetiva por parte de los pacientes demostró satisfacción en cuanto a su capacidad de inteligibilidad del habla, además de un adecuado mecanismo de deglución, ya que han podido retomar una dieta prácticamente normal.
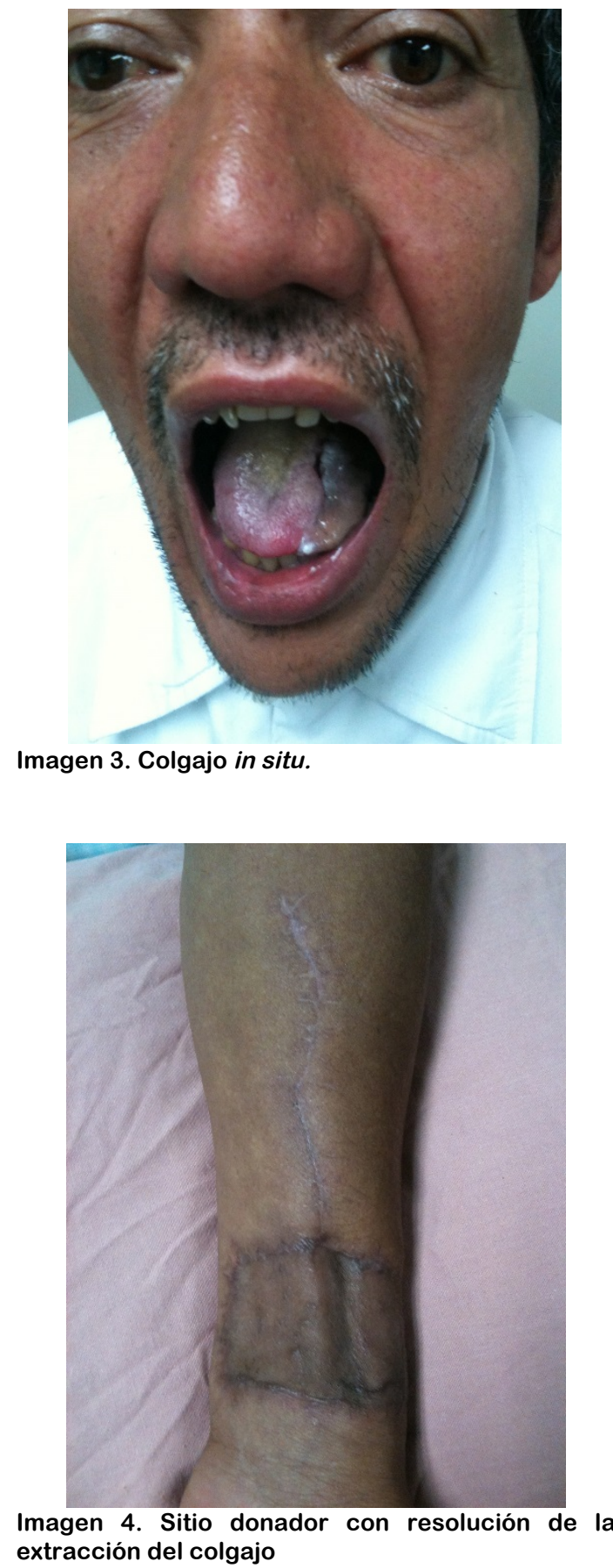

\section{DISCUSIÓN}

En la actualidad, múltiples estudios han buscado el tipo "ideal" de reconstrucción post resección en tumores de lengua, sin embargo, ninguno lo ha logrado demostrar. Los resultados más promisorios en la materia vienen en la forma de hacer refinamientos a las técnicas ya utilizadas, ade- 
más, conforme avanza la capacidad técnica y tecnológica en cuanto a las reconstrucciones microquirúrgicas, los resultados funcionales aumentarán los niveles de evidencia, tornándose en los estándares a seguir. Entre éstos, el colgajo libre radial de antebrazo corregido para la anatomía y con re-inervación es el que ha demostrado la mayor capacidad de rehabilitación funcional hasta el momento, sin embargo, existen esfuerzos por presentar otros tipos de colgajos como adecuadas formas de alcanzar el mismo resultado.

Es aquí donde la experiencia del cirujano se vuelve primordial al momento de la selección del abordaje quirúrgico, el cual independientemente de la técnica empleada debe conservar los principios básicos de la reconstrucción de lengua y buscar siempre el mejor resultado funcional para el paciente.

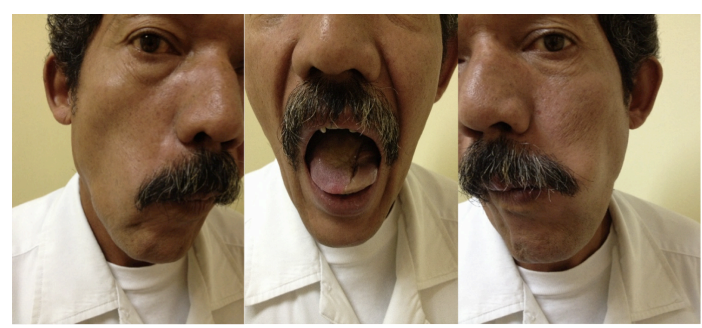

Imagen 5. Evolución de la recuperación anatómica y funcional del colgajo.

Finalmente, para apreciar la recuperación funcional en lo referente a movilidad y articulación de palabras, puede visitarse los videos respectivos mediante los enlaces

\section{http://youtu.be/OGic7mTOhH4}

\section{http://youtu.be/bxEaaXZIpMc}

\section{CONCLUSIONES Y RECOMENDACIONES}

La reconstrucción de lengua continúa siendo un gran reto quirúrgico tanto por la complejidad del procedimiento como por la gran cantidad de posibles abordajes que existen.

La evidencia actual, que aún continúa en evolución, muestra una tendencia creciente en abordajes que recuperen la mayor capacidad funcional post cirugía.
El colgajo libre radial de antebrazo continúa siendo una de las mejores alternativas de reconstrucción, para defectos mayores de lengua post resección de tumor. Este abordaje presenta gran futuro para la reconstrucción de lengua por los refinamientos realizados a la técnica original, que han demostrado mejores resultados funcionales.

La experiencia en el HSJD se asemeja a la experiencia mundial, tanto en el tipo de patología encontrada en los pacientes así como de los resultados obtenidos post cirugía. Sin embargo, es necesario hacer notar que el número de pacientes es pequeño para el periodo estudiado.

\section{REFERENCIAS BIBLIOGRÁFICAS}

1. Ariyan S. The pectoralis major myocutaneous flap. A versatile flap for reconstruction in the head and neck. Plast Reconstr Surg 1979;63:73-81.

2. Salibian AH Allison GR Rappaport I Krugman ME McMicken BL Etchepare TL. Total and subtotal glossectomy: Function after microvascular reconstruction. Plast Reconstr Surg 1990;85:513-524.

3. Lyos AT Evans GRD Perez D Schusterman MA. Tongue reconstruction: Outcomes with the rectus abdominis flap. Plast Rec Surg 1999;103:442-447.

4. Yang GF Chen BJ Gao YZ. Forearm free skin transplantation. Natl Med J China 1981;61:139-141.

5. Soutar DS Scheker LR Tanner SB McGregor IA. The radial forearm flap: $A$ versatile method for intra-oral reconstruction. Br J Plast Surg 1983;36(1):1-8.

6. Takato $\mathrm{T}$ Harii K Ebihara S. Oral and pharyngeal reconstruction using the free forearm flap. Arch Otolaryngol Head Neck Surg 1987;113:873-879.

7. Borggreven PA Leeuw IV Rinkel RN et al. Swallowing after major surgery of the oral cavity or oropharynx: a prospective and longitudinal assessment of patients treated by microvascular soft tissue reconstruction. Head Neck 2007;29(7):638-647.

8. Chong V. Oral cavity cancer. Cancer Imaging 2005;5:S49-S52.

9. Bokhari WA Wang SJ. Tongue reconstruction: recent advances. Curr Opin Otolaryngol Head Neck Surg 2007;15:202-207. 
10. Mosier K Liu WC Behin B Lee C Baredes S. Cortical adaptation following partial glossectomy with primary closure: implications for reconstruction of the oral tongue. Ann Otol Rhinol Laryngol 2005;114:681687.

11. Su WF Hsia YJ Chang YC Chen SG Sheng H. Functional Comparison after Reconstruction with a Radial Forearm Free Flap or a Pectoralis Major Flap for Cancer of the Tongue. Arch Otolaryngol Head and Neck Surgery 2003;128:412-418.

12. Biglioli F Liviero F Frigerio A Rezzonico A Brusati R. Function of the sensate free forearm flap after partial glossectomy. J Craniomaxillofac Surg 2006;34:332-339.

13. Katou F Shirai N Kamakura S et al. Intraoral reconstruction with innervated forearm flap: a comparison of sensibility and reinnervation in innervated versus noninnervated forearm flap. Oral Surg Oral Med Oral Pathol Oral Radiol Endod 1995; 80(6):638-644.

14. Lapeyre M Bollet MA Racadot S et al. Postoperative brachitherapy alone \& combined postoperative and brachitherapy boost for squamous cell carcinoma of the oral cavity, with positive or close margins. Head Neck 2004;26:216-223.

15. Chen AY Myer JN. Cancer of the oral cavity. Dis Mon. 2001;47:275-361.

\section{CONFLICTO DE INTERÉS}

En el presente trabajo no existió ningún conflicto de interés. 